\title{
Reactive intuitionistic tableaux
}

\author{
D. M. Gabbay
}

Received: 11 May 2009 / Accepted: 14 October 2009 / Published online: 2 September 2010

(C) Springer Science+Business Media B.V. 2010

\begin{abstract}
We introduce reactive Kripke models for intuitionistic logic and show that the reactive semantics is stronger than the ordinary semantics. We develop Beth tableaux for the reactive semantics.
\end{abstract}

Keywords Intuitionistic logic $\cdot$ Reactive Kripke models $\cdot$ Beth tableaux

\section{Introduction}

In Gabbay (2008) we introduced the idea of reactivity and studied reactive Kripke models for modal logics. In many subsequent papers we studied other reactive systems such as reactive argumentation frames, reactive automata, reactive grammars, reactive preferential logics, reactive contrary to duties, reactive inheritance networks, and many more.

The purpose of this paper is to introduce reactive intuitionistic frames (Kripke frames and Beth frames) and study their expressive power and properties.

We begin by briefly introducing the idea of reactive networks (including reactive Kripke models). Consider the network of Fig. 1.

Let us first ignore the double arrow in Fig. 1. Without the double arrow, Fig. 1 is a three point Kripke model for intuitionistic logic. So for example if we want to evaluate

\footnotetext{
D. M. Gabbay ( $\varangle)$

King's College London, London, UK

e-mail: dov.gabbay@kcl.ac.uk

D. M. Gabbay

Bar Ilan University Israel, Ramat Gan, Israel

D. M. Gabbay

University of Luxembourg, Luxembourg city, Luxembourg
} 
Fig. 1 E1

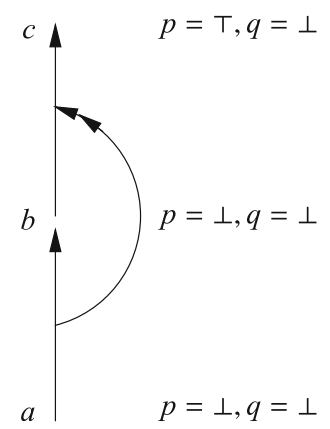

$a \not \models p \rightarrow q$, we must check whether there exists a higher point $s$ to $a$ (including $a$ itself) with $x \vDash p$ but $x \not \models q$.

The above definition is set-theoretical. The notion $x \vDash A$ is defined inductively, and the graph of Fig. 1 without the double arrow is just a graph suggesting a Kripke model with a reflexive and transitive binary relation.

To be more precise, Fig. 1 (without the double arrow) suggests a set $S=\{a, b, c\}$, a relation $R=\{(a, b),(b, c)\}$ and the reflexive and transitive closure of $R$ being $R^{*}=\{(a, a),(a, b),(a, c),(b, b),(b, c),(c, c)\}$. If we use an explicit formula for $R^{*}$, we get: $x R^{*} y$ iff $x=y$ or $x R y$ or for some $k \geq 1$ and some $t_{1}, \ldots, t_{k}$, we have $x R t_{1} \wedge t_{1} R t_{2} \wedge \ldots \wedge t_{k-1} R t_{k} \wedge t_{k} R y$.

The assignment $h$ to the atoms is also indicated in the figure.

So the Kripke model is $\left(S, R^{*}, a, h\right)$, with $h(p)=\{c\}$ and $h(q)=\varnothing$.

So to check whether $a \not \models p \rightarrow q$ we simply ask set-theoretically whether $\exists x\left(a R^{*} x\right.$ and $x \vDash p$ and $x \not \models q)$.

To introduce the reactive approach we envisage ourselves walking along the arrows of the graph from point $a$ onwards and at each point $x$ that we pass, we evaluate $x \vDash p$ and $x \vDash q$ and compare. This is an actual walk and search along the graph.

Of course, the end result is the same. If there is an $x$ such that $a R^{*} x$ and $x \vDash p$ and $x \not \models q$ then we will walk into it sooner or later and vice versa.

Now given this 'walk along the graph' point of view, the reactive double arrow makes sense. What it does is the following: As we cross from $a$ to $b$, the double arrow gets activated and disconnects the path from $b$ to $c$. So we do not get to the point $c$ where $c \vDash p$ and $c \not \models q$. Without getting to $c$, we will report that $a \vDash p \rightarrow q$ holds, because we cannot get to the counterexample, etc. So in the reactive model of Fig. 1 with the double arrow, we have $a \vDash p \rightarrow q$.

We now sum up. We introduced two ideas here.

1. Evaluation in Kripke models is done by 'walk along the arrows and check and report' policy.

2. Double arrows along the way can disconnect connections and control where we can go. ${ }^{1}$

\footnotetext{
1 There are more complex options for reactivity:

(a) Double arrows can switch arrows on and off.

(b) Double arrows can emanate from other double arrows.
} 
Fig. 2 E2

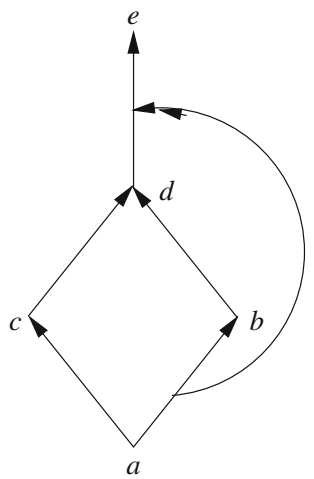

Consider now Fig. 2. In this figure, when we walk along $a \rightarrow b \rightarrow c$ we cannot continue from $d$ to $e$, because $d \rightarrow e$ gets disconnected. However, when we walk along $a \rightarrow c \rightarrow d$, we can continue to $e$ because there is no double arrow along the path.

Section 2 gives the formal definitions involved and introduces the reactive models. We also show that we get a richer semantics than ordinary Kripke models.

The idea of reactivity is a general one and can apply to Beth models as well. Beth models are like Kripke models except the inductive truth definition is different. We need the notion of an Belt anti-chain of points. Given $\left(S, R^{*}, a, h\right)$, and $t \in S$ then a set $T \subseteq S$ is a Belt anti-chain for $t$ if all points of $T$ are $R^{*}$ not comparable and every maximal $R^{*}$ chain beginning at $t$ must meet the Belt $T$.

We have $t \vDash A$ iff there exists an antichain Belt $T$ for $t$ such that for all $x \in T, x \vDash A$.

Turning a model reactive is even easier, if we give the correct definition of a reactive path. A reactive path beginning at $t$ is a trace of a walk along the arrows from $t$ onwards, where all double arrows are taken into account. So hopefully we can define reactive Beth models as well.

In Fig. 2 there are two maximal reactive paths $a \rightarrow b \rightarrow d$ and $a \rightarrow c \rightarrow d \rightarrow e$.

\section{Reactive Kripke frames}

This section introduces reactive Kripke frames for intuitionistic logic and shows that intuitionistic propositional logic is complete for such frames. We also show that there are intermediate logics which are complete for a class of reactive Kripke frames but are not complete for any class of ordinary Kripke frames.

Thus reactive Kripke frames is a richer and stronger semantics than ordinary frames.

The above also means that we can study a richer class of intermediate logics, e.g. intermediate logics generated by finite reactive frames. We shall see in Remark 3.8 what kind of Heyting like algebras one gets from finite reactive frames.

Footnote 1 continued

(c) We can have an inductively iterated version of the above.

In this paper we are keeping the reactivity simple. 
To appreciate the opportunities opening for us through the notion of reactive Kripke frames for intuitionistic logic, consider a famous beautiful theorem of L. Maksimova Maksimova (1977).

- There are only seven intermediate logics which have interpolation

Is this still true if we take into account logics generated by reactive frames? The notion of 'logic' may not be the same!

A later section will provide tableaux for logics defined by finite frames.

Definition 2.1 (Ordinary Kripke models for intuitionistic propositional logic) A Kripke model has the form $\mathbf{m}=\left(S, R, R^{*}, a, h\right)$ where $S$ is a non-empty set of worlds and $R \subseteq S \times S$ is a binary relation on $S$. $R^{*}$ is the reflexive and transitive closure of $R,{ }^{2} a \in S$ is the actual world and $h$ is an assignment, giving for each atomic $q$ a subset $h(q) \subseteq S$.

The system $\mathbf{F}=\left(S, R, R^{*}, a\right)$ is called a Kripke frame.

The following holds

$$
t \in h(q) \wedge t R^{*} s \Rightarrow s \in h(q) .
$$

The satisfaction relation is defined as follows, for $t \in S$ and a propositional formula A.

1. $t \vDash q$ iff $t \in h(q)$, for $q$ atomic

2. $t \vDash A \wedge B$ iff $t \vDash A$ and $t \vDash B$

$t \vDash A \vee B$ iff $t \vDash A$ or $t \vDash B$.

3. $t \vDash A \rightarrow B$ iff for all $s$ such that $t R^{*} s$, if $s \vDash A$ then $s \vDash B$

4. $t \vDash \neg A$ iff for all $s$ such that $t R^{*} s$ we have $s \not \models A$.

5. We say $A$ holds in the model iff $a \vDash A$.

6. Note we have not used $R$ at all, only $R^{*}$, and that models have actual worlds and satisfaction in a model is defined as satisfaction in the actual world.

This presentation is for later comparison, with the reactive case.

Theorem 2.2 Intuitionistic propositional logic is complete for the semantics of Definition 2.1.

Proof Well known result.

Definition 2.3 (Pre-reactive Kripke models) A pre-reactive Kripke model has the form $\left(S, S^{*}, R, a, h\right)$ where $S$ is a non-empty set, $a \in S, R \subseteq S \times S$ is a binary relation (not necessarily reflexive nor transitive) and $S^{*}$ is the set of all $R$ increasing sequences $\beta$ of elements from $S$ of the form $\beta=\left(a_{0}, a_{1}, \ldots, a_{n}\right)$ such that $a_{0}=a$ and for $i=0, \ldots, n-1$ we have $a_{i} R a_{i+1}$. We denote $a_{n}$ by $|\beta| . h$ is an assignment giving for each atomic $q$ a subset $h(q) \subseteq S$ such that

$$
t \in h(q) \wedge t R s \Rightarrow s \in h(q)
$$

$\overline{2 \text { We have } x R^{*} y \text { iff } x=y \text { or } x R y}$ or $\exists t_{1}, \ldots, t_{m}\left(x R t_{1} \wedge t_{1} R t_{2} \wedge \ldots t_{m-1} R t_{m} \wedge t_{m} R y\right)$, for some $m \geq 1$. 
We define satisfaction for $\beta$, a sequence in $S^{*}$, as follows. (We need the notion of: $\beta^{\prime}$ is an extension of $\beta$ iff $\beta$ is an initial sequence of $\beta^{\prime}$, i.e. $\beta=\left(a, t_{1}, \ldots, t_{k}\right)$ and $\left.\beta^{\prime}=\left(a, t_{1}, \ldots, t_{k+n}\right) n \geq 0\right)$.

1. $\beta \vDash q$ iff $|\beta| \in h(q)$, for $q$ atomic.

2. $\beta \vDash A \wedge B$ iff $\beta \vDash A$ and $\beta \vDash B$.

$\beta \vDash A \vee B$ iff $\beta \vDash A$ or $\beta \vDash B$.

3. $\beta \vDash A \rightarrow B$ iff for every extension $\beta^{\prime}$ of $\beta$, we have that if $\beta^{\prime} \vDash A$ then $\beta^{\prime} \vDash B$.

4. $\beta \vDash \neg A$ iff for all extension $\beta^{\prime}$ of $\beta$ we have $\beta^{\prime} \not \models A$.

5. $A$ holds in the model if $a \vDash A$.

Lemma 2.4 Let $\mathbf{m}=\left(S, S^{*}, R, a, h\right)$ be a model. Let $R^{*}$ be the reflexive and transitive closure of $R$. Consider the model $\mathbf{n}=\left(S, R, R^{*}, a, h\right)$. Then we have for every $\beta \in S^{*}$

$$
\beta \vDash A \text { in } \mathbf{m} \text { iff }|\beta| \vDash A \text { in } \mathbf{n} \text {. }
$$

Proof By induction on $A$. The crucial point is $A \rightarrow B$.

1. Assume $\beta \vDash A \rightarrow B$. Then for all $\beta^{\prime}$ extending $\beta$ we have that $\beta^{\prime} \vDash A$ implies $\beta^{\prime} \vDash B$.

By the induction hypothesis we have if $\left|\beta^{\prime}\right| \vDash A$ then $\left|\beta^{\prime}\right| \vDash B$. We now show $|\beta| \vDash A \rightarrow B$.

Let $s$ be such that $|\beta| R^{*} s$, then $|\beta|=s$ or $|\beta| R s$ or there exist $s_{0}, s_{1}, \ldots, s_{m}$ such that $s_{0}=|\beta|$ and $s_{m}=s$ and for $i=0, \ldots, m-1$ we have $s_{i} R s_{i+1}$. Hence $\beta^{\prime}=\beta *\left(s_{1}, \ldots, s_{m}\right)$ is an extension of $\beta$ with $\left|\beta^{\prime}\right|=s_{m}=s$. Therefore if $s \vDash A$ then $\left|\beta^{\prime}\right| \vDash A$, hence $\beta^{\prime} \vDash A$ by the induction hypothesis, hence $\beta^{\prime} \vDash B$, hence $\left|\beta^{\prime}\right| \vDash B$, i.e. $s \vDash B$.

2. Now assume $|\beta| \vDash A \rightarrow B$. Let $\beta^{\prime}$ extend $\beta$. Hence $|\beta| R^{*}\left|\beta^{\prime}\right|$. So if $\beta^{\prime} \vDash A$ then $\left|\beta^{\prime}\right| \vDash A$ hence $\left|\beta^{\prime}\right| \vDash B$ hence $\beta^{\prime} \vDash B$.

The proof for $\neg A$ is similar.

Remark 2.5 The second type of model is easier to turn reactive. In this new type of model, we view the evaluation of $A \rightarrow B$ at a node $t$ as 'going along the relation $R$ and at whatever point $t^{\prime}$ we reach, if $t^{\prime} \vDash A$ then $t^{\prime} \vDash B$.' So in this definition we actually have to traverse the arcs of the model.

Note that $R$ needs not be reflexive nor transitive. We get these properties from the evaluation process. So consider Figs. 3 and 4.

Figure 3 gives $S_{1}=\{t, s\}, R_{1}=\{(t, s)\}$. Figure 4 gives $S_{2}=\{t, s\}, R_{2}=\{(t, t),(t, s)\}$. We have $S_{1}^{*}=\{(t),(t, s)\}$ and $S_{2}^{*}=\{(t),(t, \ldots \ldots, t, s) \mid m=0,1,2, \ldots\} . S_{1}^{*}$ corresponds to the ordinary Kripke model with two linear points $1<2$ (as in Fig. 3) and $S_{2}^{*}$ corresponds to the ordinary Kripke model with the infinite comb of Fig. 5 such that the assignments to the points in $\{0,1, \ldots\}$ are all identical (representing the point $t$ and also the assignment to the points in $\left\{w_{0}, w_{1}, w_{2}, \ldots, w_{n} \ldots\right\}$ are all identical, (representing the point $s$ ). 


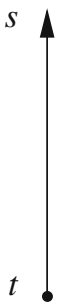

Fig. 3 A1

Fig. 4 A2

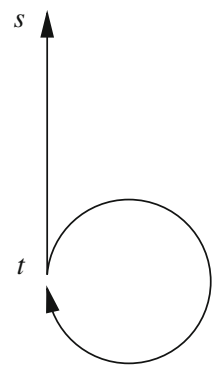

Fig. 5 A3

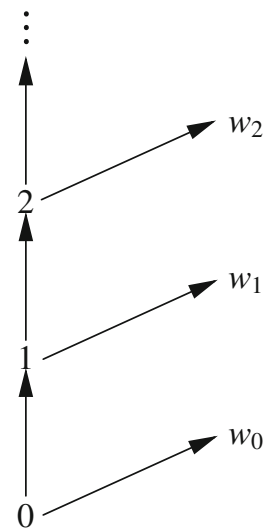

Definition 2.6 (Reactive intuitionistic Kripke frame) A reactive intuitionistic Kripke frame has the form $(S, \mathbb{R}, a)$, where $a \in S$ and $\mathbb{R}$ is a set of pairs of the form

1. $(x, y) \in S \times S$ called arrows

2. $((x, y),(u, v)) \in S^{2} \times S^{2}$ called reactive double arrows.

Example 2.7 A reactive frame, see Fig. 6.

We have $\mathbb{R}=\{(t, s),(t, t),((t, t),(t, s))\}$ and $a=t$

Definition 2.8 (How reactivity operates)

1. Let $\mathbf{m}=(S, \mathbb{R}, a)$ be a frame. Let $(t, s) \in \mathbb{R}$ be an arrow. Define $\mathbb{R}_{(t, s)}$ to be $\mathbb{R}_{(t, s)}=\mathbb{R}-\{(x, y) \mid((t, s),(x, y)) \in \mathbb{R}\} . \mathbb{R}_{(t, s)}$ is the result of traversing the arc 
Fig. 6 A5

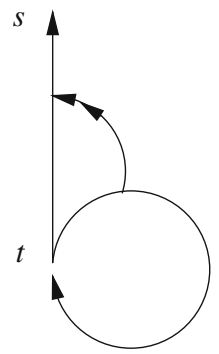

$t \rightarrow s$ and cancelling all connections as indicated by double arrows emanating from $t \rightarrow s$.

2. Let $\beta=\left(a, t_{1}, \ldots, t_{k}\right)$. We now define $\mathbb{R}_{\beta}$ by induction on $k$. For $k=1$, we let $\mathbb{R}_{\beta}=\mathbb{R}_{\left(a, t_{1}\right)}$, provided $\left(a, t_{1}\right) \in \mathbb{R}$.

Assume $\mathbb{R}_{\beta}$ has been defined for $\beta=\left(a, t_{1}, \ldots, t_{k}\right)$ and assume $\left(t_{k}, t_{k+1}\right) \in \mathbb{R}_{\beta}$.

Define $\mathbb{R}_{\beta^{\prime}}$ for $\beta^{\prime}=\left(a, t_{1}, \ldots, t_{k+1}\right)$ to be $\mathbb{R}_{\beta^{\prime}}=\left(\mathbb{R}_{\beta}\right)_{\left(t_{k}, t_{k+1}\right)}$.

3. Let $\mathbb{R}_{\beta}$ be a reactive relation as defined in (2) where $\beta=\left(a, t_{1}, \ldots, t_{k}\right)$.

Let

$$
\begin{aligned}
& \beta_{0}=\beta \\
& \beta_{1}=\left(a, t_{1}, \ldots, t_{k+1}\right) \\
& \vdots \\
& \beta_{n}=\left(a, t_{1}, \ldots, t_{k+n}\right)
\end{aligned}
$$

We say $\beta_{n}$ for $n \geq 0$ is a legitimate extension of $\beta$ iff $n=0$ or $n \geq 1$ and the following holds.

- $\left(t_{k}, t_{k+1}\right) \in \mathbb{R}_{\beta}$

- $\mathbb{R}_{\beta_{1}}$ is obtained from $\mathbb{R}_{\beta}$ as in (1) above

- $\left(t_{k+n-1}, t_{k+n}\right) \in \mathbb{R}_{\beta_{n-1}}$

- $\mathbb{R}_{\beta_{n}}$ is obtained from $\mathbb{R}_{\beta_{n-1}}$ as in (1) above.

4. We say $\beta_{1}$ is an immediate legitimate extension of $\beta$.

Lemma 2.9 Let $\left(S, \mathbb{R}_{(a)}\right.$, a) be a reactive Kripke frame. Let $\beta$ be a legitimate extension of $(a)$, and $\beta \neq(a)$. Write $\beta=\left(a, t_{1}, \ldots, t_{k}\right)$. Then we have aRt $t_{1}, t_{1} R t_{2}, \ldots$, $t_{k-1} R t_{k}$, where $R=\{(x, y) \mid(x, y) \in S \times S \cap \mathbb{R}\}$.

Proof By induction on $k$. For $k=1$ we do have $a R t_{1}$.

We see from the construction of any $\mathbb{R}_{\beta}$ that we have $\mathbb{R}_{\beta} \subseteq \mathbb{R}$.

Hence if $\beta^{\prime}=\beta *\left(t_{k+1}\right)$ with $|\beta|=t_{k}$ and $\left(t_{k}, t_{k+1}\right) \in \mathbb{R}_{\beta}$, then we have $t_{k}$ $R t_{k+1}$.

\section{Definition 2.10 (Satisfaction in a reactive model)}

1. Let $(S, \mathbb{R}, a)$ be a reactive frame. Let $R=\{(x, y) \mid x, y \in S$ and $(x, y) \in \mathbb{R}\}$. Let $h$ be an assignment such that $t \in h(q)$ and $t R s$ implies $s \in h(q)$. 
Let $\beta$ be a legitimate extension of $(a)$. Let $\mathbb{R}_{\beta}$ be the corresponding relation. Let $\mathbf{m}_{\beta}=\left(S, \mathbb{R}_{\beta},|\beta|, h\right)$. Note that if $\gamma=\left(|\beta|, t_{1}, \ldots, t_{m}\right)$ is a legitimate extension of $|\beta|$ in $\mathbf{m}_{\beta}$, then $\beta^{1}=\beta *\left(t_{1}, \ldots, t_{m}\right)$ is a legitimate extension of (a) in $(S, \mathbb{R}, a)$.

2. We define satisfaction as follows:

- $\beta \vDash q$ iff $|\beta| \in h(q)$, for $q$ atomic

- $\beta \vDash A \wedge B$ iff $\beta \vDash A$ and $\beta \vDash B$

- $\beta \vDash A \vee B$ iff $\beta \vDash A$ or $\beta \vDash B$

3. $\beta \vDash A \rightarrow B$ in $\mathbf{m}_{\beta}$ iff for every legitimate extension $\beta^{\prime}$ of $\beta$, if $\beta^{\prime} \vDash A$ in $\mathbf{m}_{\beta^{\prime}}$ then $\beta^{\prime} \vDash B$ in $\mathbf{m}_{\beta^{\prime}}$.

4. $\beta \vDash \neg A$ in $\mathbf{m}_{\beta}$ iff for all legitimate extensions $\beta^{\prime}$ of $\beta$ we have $\beta^{\prime} \not \models A$ in $\mathbf{m}_{\beta^{\prime}}$.

5. We say $(S, \mathbb{R}, a, h) \vDash A$ iff $(a) \vDash A$, (in words: $A$ holds in the model).

Lemma 2.11 Let $(S, \mathbb{R}, a, h)$ be a model and assume that $\beta \vDash A$ in $\mathbf{m}_{\beta}$ and that $\beta^{\prime}$ is a legitimate extension of $\beta$, then $\beta^{\prime} \vDash A$ in $\mathbf{m}_{\beta^{\prime}}$.

Proof By induction on $A$.

1. For $A$ atomic, this follows from a previous Lemma 2.9 .

2. The cases of $\wedge$ and $\vee$ are immediate.

3. Assume $\beta \vDash A \rightarrow B$, then for any legitimate extension $\beta^{\prime}$ of $\beta$, if $\beta^{\prime} \vDash A$ in $\mathbf{m}_{\beta^{\prime}}$ then $\beta^{\prime} \vDash B$ in $\mathbf{m}_{\beta^{\prime}}$.

But now since any legitimate extension $\beta^{\prime \prime}$ of $\beta^{\prime}$ is also a legitimate extension of $\beta$ we get that $\beta^{\prime} \vDash A \rightarrow B$ in $\mathbf{m}_{\beta^{\prime}}$.

4. The case of $\neg$ is similar.

Example 2.12 (Satisfaction in the frame of Fig. 6) Let $q$ be atomic. Let $h(q)$ be $\{s\}$. Then

1. $(t) \not \models q$

2. $(t) \not \models \neg q$

3. $(t) \not \models \neg q \rightarrow q$.

(1) and (2) are clear. To show (3), note that $\beta=(t, t)$ is a legitimate extension of $(t)$ and $\mathbb{R}_{(t, t)}$ is

$$
\{(t, t),((t, t),(t, s)\} .
$$

In $\left(S, \mathbb{R}_{(t, t)},(t, t)\right.$ we have $(t, t) \vDash \neg q$ but $(t, t) \not \models q$.

Lemma 2.13 The logic defined by reactive satisfaction is intuitionistic logic. ${ }^{3}$

Proof

1. Since ordinary pre-reactive models are reactive models, (by Definition 2.3 and Lemma 2.4) the logic is not stronger than intuitionistic logic.

\footnotetext{
${ }^{3}$ Let $\mathbb{K}$ be a class of reactive frames. Let $A$ be a wff which holds in any frame in the class under any assignment. Let $\operatorname{Logic}(\mathbb{K})$ be the set of all such wffs $A$. Then $\operatorname{Logic}(\mathbb{K})$ is a 'logic'. All its wffs are classical theorems but this 'logic' may not be closed under substitution.
} 
2. From Lemma 2.11 we see the logic is not weaker either.

Theorem 2.14 The logic of the frame Fig. 6 is not complete for any class of ordinary intuitionistic Kripke frames.

Proof The proof has four parts, (A)-(D).

(A)

(A) The following formula holds in the frame of Fig. 6, under any $h$,

(a) $x \vee(x \rightarrow(q \vee \neg q)), x, q$ atomic

(b) $(x \rightarrow y) \vee(y \rightarrow x), x, y$ atomic.

We check (1).

If $x=\perp$ at $(t)$ then to falsify $x \rightarrow(q \vee \neg q)$ we need to go to $(t, s)$ where $x$ can hold. We cannot go to $(t, t)$ because $(t, t) \not \models x$. At $(t, s)$ clearly $q \vee \neg q$ holds.

We check (2).

Assume $x \rightarrow y$ is false at $(t)$. Then we have either

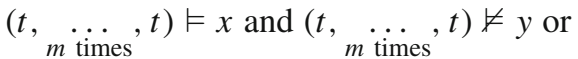

$(t, \underset{n \text { times }}{\ldots,} t, s) \vDash x$ and $(t, \underset{n \text { times }}{\ldots}, t, s) \not \models y, m, n \geq 0$.

In the first case, we have $(t) \vDash x$ and hence $(t) \vDash y \rightarrow x$.

In the second case we have $(t, s) \vDash x,(t, s) \not \models y$. Hence $(t) \vDash \neg y$ and so $(t) \vDash y \rightarrow x$.

(B) We now show that any ordinary frame which satisfies (1) and (2) under any $h$ must be either a single point or the frame of Fig. 3 .

This is well known because otherwise either (1) or (2) can be falsified. (1) is falsified by a 3 point chain and (2) by a two point anti-chain. So we can have neither.

(C) We now show that in Fig. 3 or in a single point (3) must hold.

(a) $x \vee \neg x \vee(\neg x \rightarrow x)$.

To falsify $x \vee \neg x$ we need Fig. 3 with $t \not \models x$ and $s \vDash x$ but from the latter it follows that $t \vDash \neg \neg x$ holds and hence $t \vDash \neg x \rightarrow x$.

(D) Our proof is concluded because Example 2.12 shows that (3) can be falsified in the frame of Fig. 6.

Remark 2.15 It is helpful to have another view of Fig. 6. The frame has two paths, as in Fig. 7

We can view Fig. 7 as an ordinary 3 point Kripke model with the understanding that the assignment at $t$ and $(t, t)$ is the same, i.e. for every $q, t \in h(q)$ iff $(t, t) \in h(q)$.

This is common to reactive models, that they can be 'unfolded' as models of paths with restrictions on the assignments. Gabbay and Marcelino (2009) studies such models. We examine this notion in the next section.

\section{Folding reactive frames}

We saw in the last section that the reactive frame of Fig. 6 can be unfolded into the ordinary frame of Fig. 7, with the added understanding that the points $(t)$ and $(t, t)$ 
Fig. 7 A6

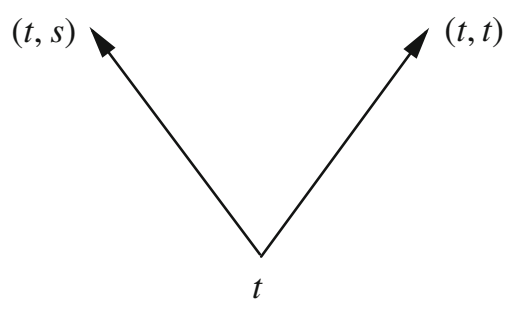

must give the same values to the atoms. This unfolding process can be done in a systematic manner, and it seems to have significance for developing Beth tableaux for reactive intuitionistic logics. So in this section we study it in detail. We are going to unfold and then fold again.

Let $(S, \mathbb{R}, a)$ be a reactive frame. Let $\beta$ be a legitimate path of the form $\beta=$ $\left(a, t_{1}, \ldots, t_{k}\right)$. We saw that we can calculate $\mathbb{R}_{\beta} \subseteq \mathbb{R}$. Let $\mathbf{F}_{\beta}=\left(S, \mathbb{R}_{\beta},|\beta|\right)$.

If $S$ is finite and $\beta$ ranges over all legitimate paths, we get only a finite number of different frames $\mathbf{F}_{\beta}$. Let us take advantage of this.

Definition 3.1 (Path equivalence relation) Let $\mathbf{m}=(S, \mathbb{R}, a, h)$ be a reactive model. Define an equivalence relation on the paths of the model as follows:

- $\beta \equiv \gamma$ iff $|\beta|=|\gamma|$ and $\mathbb{R}_{\beta}=\mathbb{R}_{\gamma}$.

Let $\Omega$ be the set of equivalence classes, $\{\beta|\equiv| \beta$ a legitimate path extending $(a)\}$.

Then $\Omega$ is finite.

Define $\rho$ (respectively $\rho_{1}$ ) on $\Omega$ as follows:

- $\beta / \equiv \rho \gamma / \equiv$ (respectively: $\beta / \equiv \rho_{1} \gamma / \equiv$ ) iff for some $\beta_{1} \equiv \beta$ and $\gamma_{1} \equiv \gamma$ we have $\gamma_{1}$ is a legitimate extension (respectively: an immediate legitimate extension) of $\beta_{1}$.

Lemma 3.2 $\rho$ is reflexive and transitive, and is the reflexive and transitive closure of $\rho_{1}$.

Proof Reflexivity is not a problem. We show transitivity.

Claim If $\beta_{1}$ is a legitimate extension of $\alpha_{1}$ and $\beta_{1} \equiv \beta_{2}$ and $\gamma_{2}$ is a legitimate extension of $\beta_{2}$, then there exists a $\gamma_{1} \equiv \gamma_{2}$ such that $\gamma_{1}$ is a legitimate extension of $\alpha_{1}$.

We now prove the claim:

1. Since $\beta_{1}$ is a legitimate extension of $\alpha_{1}$, we have that $\beta_{1}=\alpha_{1}$ or for some $k \geq 1$, we have $\beta_{1}=\alpha_{1} *\left(t_{1}, \ldots, t_{k}\right)$ where $\left|\alpha_{1}\right| R t_{1} \wedge t_{1} R t_{2} \wedge \ldots t_{k-1} R t_{k}$ and $\left|\beta_{1}\right|=t_{k}$.

2. Since $\beta_{1} \equiv \beta_{2}$ we get that $\mathbb{R}_{\beta_{1}}=\mathbb{R}_{\beta_{2}}$.

3. Since $\gamma_{2}$ is a legitimate extension of $\beta_{2}$, we have $\gamma_{2}=\beta_{2}$ or for some $m \geq 1$ we also have $\left(s_{1}, \ldots, s_{m}\right)$ such that $\gamma_{2}=\beta_{2} *\left(s_{1}, \ldots, s_{m}\right)$ and $\left|\beta_{2}\right| R s_{1} \wedge s_{1} R s_{2} \wedge$ $\ldots s_{m-1} R s_{m}$ and $\left|\gamma_{2}\right|=s_{m}$.

Consider the path

$$
\gamma_{1}=\alpha_{1} *\left(t_{1}, \ldots, t_{k}\right) *\left(s_{1}, \ldots, s_{m}\right) .
$$


where if $\beta_{1}=\alpha_{1}$ then $\left(t_{1}, \ldots, t_{k}\right)$ does not appear and if $\gamma_{2}=\beta_{2}$ then $\left(s_{1}, \ldots\right.$, $s_{m}$ ) does not appear in $\gamma_{1}$.

It is clear that $\left|\gamma_{1}\right|=\left|\gamma_{2}\right|$.

We want to show that

$$
\mathbb{R}_{\gamma_{1}}=\mathbb{R}_{\gamma_{2}}
$$

Observe that $\mathbb{R}_{\beta_{1}}=\mathbb{R}_{\beta_{2}}$. Since $\gamma_{1}$ is an extension of $\beta_{1}$ along the sequence of nodes $\left(s_{1}, \ldots, s_{m}\right)$ and $\gamma_{2}$ is the extension of $\beta_{2}$ along the sequence $\left(s_{1}, \ldots, s_{m}\right)$ (same sequence) and they both start at $\left|\beta_{1}\right|=\left|\beta_{2}\right|$ with $\mathbb{R}_{\beta_{1}}=\mathbb{R}_{\beta_{2}}$, then they end up at the same relation, namely $\mathbb{R}_{\gamma_{1}}=\mathbb{R}_{\gamma_{2}}$. Hence $\gamma_{1} \equiv \gamma_{2}$.

We now finish the proof of Lemma 3.2:

4. Since $\gamma_{1} \equiv \gamma_{2}$ and $\gamma_{1}$ extends $\alpha_{1}$, we get $\alpha_{1} / \equiv \rho \gamma_{2} / \equiv$

5. Clearly $\rho$ is the reflexive and transitive closure of $\rho_{1}$.

Lemma 3.3 Let $\mathbf{m}=(S, \mathbb{R}, a, h)$ and let $\equiv, \Omega, \rho$ be as in Definition 3.1. Consider $\mu=(\Omega, \rho, a / \equiv, \mathbf{h})$, as an ordinary Kripke model, where $\mathbf{h}$ is defined by

$$
\alpha / \equiv \in \mathbf{h}(q) \text { iff }|\alpha| \in h(q) .
$$

Then for any A we have:

$$
\alpha \vDash A \text { in } \mathbf{m} \text { iff } \alpha / \equiv \vDash A \text { in } \mu \text {. }
$$

Proof By induction on $A$.

1. For $q$ atomic this holds by the definition of $\equiv$.

2. The key case is that of $\rightarrow$.

Assume $\alpha \not \models A \rightarrow B$, then for some $\beta$ which is a legitimate extension of $\alpha$ we have $\beta \vDash A$ and $\beta \not \models B$. But we also have in this case that $\alpha / \equiv \rho \beta / \equiv$ and by the induction hypothesis, $\beta / \equiv \vDash A$ and $\beta / \equiv \not \models B$.

Now assume $\alpha / \equiv \not \models A \rightarrow B$. Then for some $\gamma / \equiv$ we have $\alpha / \equiv \rho \gamma / \equiv$ and $\gamma / \equiv \vDash A$ and $\gamma / \equiv \not \models B$. Therefore for some $\alpha_{1} \equiv \alpha$ and $\gamma_{1} \equiv \gamma$ we have $\gamma_{1}=\alpha_{1} *\left(t_{1}, \ldots, t_{k}\right)$ and $\gamma_{1}$ is a legitimate extension of $\alpha_{1}$. Hence since $\gamma_{1} \equiv \gamma$, we get $\gamma_{1} / \equiv \vDash A$ and $\gamma_{1} / \equiv \not \models B$. By the induction hypothesis we have $\gamma_{1} \vDash A$ and $\gamma_{1} \not \models B$.

Now look at the two models $\mathbf{m}_{\alpha}=\left(S, \mathbb{R}_{\alpha},|\alpha|\right)$ and $\mathbf{m}_{\alpha_{1}}=\left(S, \mathbb{R}_{\alpha_{1}},\left|\alpha_{1}\right|\right)$. Since $\alpha_{1} \equiv \alpha$, these two models are the same. So having $\gamma_{1}=\alpha_{1} *\left(t_{1}, \ldots, t_{k}\right)$ with $\gamma_{1} \vDash A$ and $\gamma_{1} \not \models B$ in $\mathbf{m}_{\gamma_{1}}$ implies that for $\delta=\alpha *\left(t_{1}, \ldots, t_{k}\right)$ we also have $\delta \vDash A$ adn $\delta \not \models B$ in $\mathbf{m}_{\alpha}$. Hence $\alpha \not \models A \rightarrow B$ in $\mathbf{m}$.

Corollary 3.4 The proof of Lemma 3.2 actually gives us a stronger result. Suppose we have a situation as described in Fig. 8 below, then there exist sequences of points from $S$ of the form 
Fig. 8 Unfolded reactive model

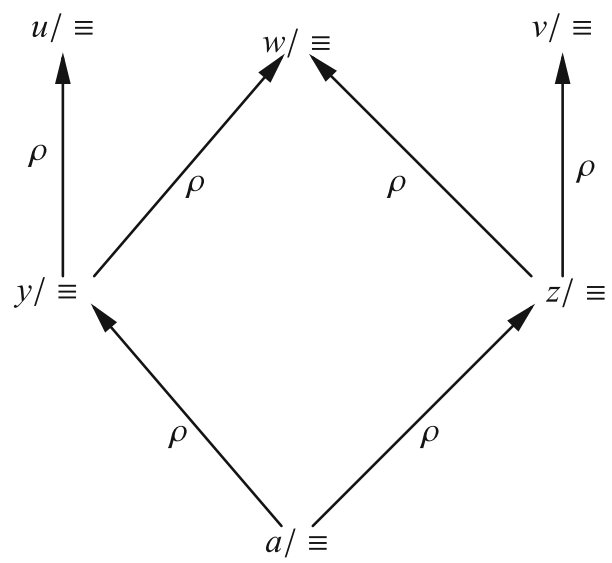

$$
\begin{aligned}
\left(a, t_{1}^{y}, \ldots, t_{k(y)}^{y}\right) & =\alpha(y) \\
\left(a, t_{1}^{z}, \ldots, t_{k(z)}^{z}\right) & =\alpha(z) \\
\left(t_{1}^{u}, \ldots, t_{k(u)}^{u}\right) & =\alpha(u) \\
\left(t_{1}^{w}, \ldots, t_{k(w)}^{w}\right) & =\alpha(w) \\
\left(s_{1}^{w}, \ldots, s_{m(w)}^{w}\right) & =\beta(w) \\
\left(t_{1}^{v}, \ldots, t_{k(v)}^{v}\right) & =\alpha(v)
\end{aligned}
$$

such that

$$
\begin{aligned}
& \alpha(y), \alpha(z), \alpha(y) * \alpha(u), \\
& \alpha(z) * \alpha(v), \alpha(y) * \alpha(w), \\
& \alpha(z) * \beta(w)
\end{aligned}
$$

are all legitimate extensions of (a) and we have

$$
\begin{aligned}
\alpha(y) & \equiv y \\
\alpha(z) & \equiv z \\
\alpha(y) * \alpha(u) & \equiv u \\
\alpha(z) * \alpha(v) & \equiv v \\
\alpha(y) * \alpha(w) & \equiv w \\
\alpha(z) * \beta(w) & \equiv w
\end{aligned}
$$

In other words, Fig. 8 from $\mu$ can be realised by actual continuous legitimate paths in $\mathbf{m}$.

In fact, we can get a general result.

Define in $\mu=(\Omega, \rho, a / \equiv, \mathbf{h})$ the following immediate successor relation $\rho_{11}$ : 
- $x / \equiv \rho_{11} y / \equiv$ iff $x / \equiv \rho \quad y / \equiv$ and $x \not \equiv y$ and for all $z / \equiv$, if $s / \equiv \rho z / \equiv$ and $z / \equiv \rho \quad y / \equiv$, then either $z \equiv x$ or $z \equiv y$.

Since $x / \equiv \rho y / \equiv$ means that for some $\left(t_{1}, \ldots, t_{k}, s_{1}, \ldots, s_{m}\right)$ we have $\alpha=$ $\left(a, t_{1}, \ldots, t_{k}\right) \equiv x$ and $\beta=\left(a, t_{1}, \ldots, t_{k}, s_{1}, \ldots, s_{m}\right) \equiv y$. The condition $\alpha / \equiv$ $\rho_{11} \beta / \equiv$ means that each $\beta_{j}=\alpha *\left(s_{1}, \ldots, s_{j}\right)$ for $1 \leq j \leq m$, is either $\equiv$ equivalent to $\alpha$ or to $\beta$. Since $\alpha \neq \equiv \beta$, each $s_{i}$ is either $t_{k}$ or $s_{m}$.

Note that $\rho_{1}$ and $\rho_{11}$ are different relations. $\rho_{1}$ is defined on legitimate sequences of $\Omega$ while $\rho_{11}$ is defined on $\Omega / \equiv$. We may have that $x \rho_{1} y$ holds but $x / \equiv \rho_{11} y / \equiv$ does not hold. However, if we have $x / \equiv \rho_{11} \quad y / \equiv$ then for some $x^{\prime} \equiv x, y^{\prime} \equiv y$ we have $x^{\prime} \rho_{1} y^{\prime}$ holds.

The general result we get is the following:

Let $\mu_{0}=\left(\Omega_{0}, \rho_{11}\left\lceil\Omega_{0}, a / \equiv\right)\right.$ be any finite submodel of $\Omega$ with $\rho_{11}$ restricted to $\mu_{0}$. Then the model $\mu_{0}$ can be realised by actual sequences from $\mathbf{m}$.

Remark 3.5 (Folding reactive frames) We started with a reactive model $\mathbf{m}=$ $(S, \mathbb{R}, a, h)$ and converted it to a special model $\mu=(\Omega, \rho, a / \equiv, \mathbf{h})$. This model is special and we want to highlight some of its properties.

First note that if $\mathbf{m}$ is finite then we can effectively derive $\mu$ from $\mathbf{m}$ in tractable time. This observation is important because we shall use $\mu$ to develop Beth tableaux for $\mathbf{m}$.

We now examine $\mu$ more closely.

The elements of $\Omega$ are equivalence classes of legitimate sequences $\beta$ of $\mathbf{m}$. We have

- $\alpha \equiv \beta$ iff $|\alpha|=|\beta|$ and $\mathbb{R}_{\alpha}=\mathbb{R}_{\beta}$.

Let $\mathbf{m}=(S, \mathbb{R}, a, h)$. Let $\mathbb{T}$ be the set of all legitimate sequences of $\mathbf{m}$. We define two equivalence relations on $\mathbb{T}$.

- $\alpha \approx \beta$ iff $|\alpha|=|\beta|$

- $\alpha \approx_{\mathbb{R}} \beta$ iff $\mathbb{R}_{\alpha}=\mathbb{R}_{\beta}$

The relation $\equiv$ is the intersection of the above two relations. So if $\mathbb{E}_{1}, \ldots, \mathbb{E}_{k}$ are all the $\approx$ equivalence subsets of $\mathbb{T}$ and $\mathbb{D}_{1}, \ldots, \mathbb{D}_{m}$ are all the $\approx_{\mathbb{R}}$ equivalence subsets of $\mathbb{T}$, then the equivalence subsets of $\equiv$ are all the different combinations of the form $\mathbb{T}_{i, j}=\mathbb{E}_{i} \cap \mathbb{D}_{j} i=1, \ldots, j=1, \ldots, m$.

Actually, if $S=\left\{s_{1}, \ldots, s_{k}\right\}$ then we can have

$$
\mathbb{E}_{i}=\left\{\alpha|| \alpha \mid=s_{i}\right\}
$$

We have

$$
\Omega=\left\{\mathbb{T}_{i, j} \mid \begin{array}{c}
i=1, \ldots, k \\
j=1, \ldots, m
\end{array}\right\}
$$

Let us now look at the class $\mathbb{E}_{i}$, for fixed $i$. Let

$$
\alpha=\left(a, t_{1}, \ldots, t_{r}, e\right) \text { and } \beta=\left(a, s_{1}, \ldots, s_{r}, e\right)
$$


be two elements of this class. We have $|\alpha|=|\beta|=e \in S$. Let us ask: Are $\alpha, \beta$ also in the same class $\mathbb{D}_{j}$ for some $j$ ? Well maybe they are, depending on whether $\mathbb{R}_{\alpha}=\mathbb{R}_{\beta}$. We can regard $\approx$ also as an equivalence relation on $\Omega=\left\{\mathbb{T}_{i, j}\right\}$ :

- $\mathbb{T}_{i, j} \approx \mathbb{T}_{m, n}$ iff $i=m$.

This means that for any $\alpha \in \mathbb{T}_{i, j}, \beta \in \mathbb{T}_{m, n}$ we have $|\alpha|=|\beta|$.

But $|\alpha|=|\beta|$ means $\mathbf{h}(\alpha)=\mathbf{h}(\beta)$, since $\mathbf{h}(\alpha)=h(|\alpha|)$ and $\mathbf{h}(\beta)=h(|\beta|)$.

We can now view the model $\mu$ with $\approx$ as a model of the form

$$
\mu=(\Omega, \rho, \approx, a / \equiv, \mathbf{h})
$$

with an equivalence relation $\approx$ on $\Omega$ and the commitment:

$$
x \approx y \rightarrow \mathbf{h}(x)=\mathbf{h}(y) .
$$

This model satisfies special properties, because we know that $\mu=(\Omega, \rho, a / \equiv, \approx)$ arises from a model $\mathbf{m}=(S, \mathbb{R}, a)$ through a special construction.

We would like to list these special properties so that we can prove that any model $\mu$ with these properties comes from some reactive model $\mathbf{m}$.

We believe that this is possible to do.

Conjecture 3.6 It is possible to give conditions on $\rho$ and $\approx$ of a folded Kripke model which ensure it is equivalent to a reactive Kripke model (without $\approx$ ).

Example 3.7 (Fig. 6) We saw that the reactive Kripke frame of Fig. 6 can be presented as the folded Kripke frame of Fig. 7. We have in Fig. 7:

$$
\begin{aligned}
\Omega & =\{t, s,(t, t)\} \\
\rho & =\{(t, t),(t, s),(s, s),(t,(t, t)),((t, t),(t, t))\}
\end{aligned}
$$

and we have $t \approx(t, t)$.

Remark 3.8 Let us see what is the status of folded Kripke frames in terms of Heyting algebras. An ordinary Kripke frame $\mathbf{m}=(\Omega, \rho)$ gives rise to a free Heyting algebra $\mathbf{H}_{\mathbf{m}}$. When we add an equivalence relation $\approx$ to form $\mu=(\Omega, \rho, \approx)$ we are adding some equalities among the free generators of $\mathbf{H}_{\mathbf{m}}$. These equalities generate a congruence relation $\approx$ on $\mathbf{H}_{\mathbf{m}}$. If we let $\mathbf{H}_{\mu}=\mathbf{H}_{\mathbf{m}} / \approx$ then we get the algebra corresponding to $\mu$. It is not a free algebra.

\section{Reactive tableaux}

We begin by explaining the intuitive idea of tableaux for reactive logics. Consider the tableau of Fig. 9

The label of the tableau is $t$. This is usually the name of the possible world we are dealing with. $A$ is on the left and so we want to make $t \vDash A$ and $C \rightarrow D$ is on the right hand side of the tableau, so we want to make $t \not \models C \rightarrow D$. 
Fig. 9 tab 1

$t:$

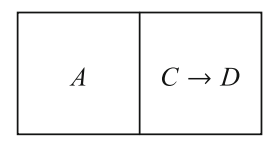

Fig. 10 tab2

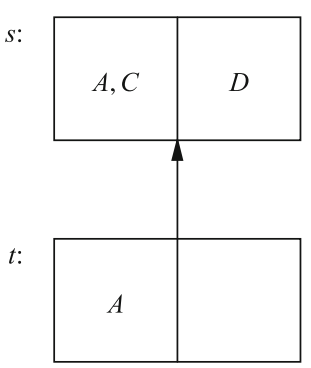

To do the latter we need an accessible world $s$. such that $t R^{*} s$ and $s \vDash A$ and $s \not \models B$. This means that we move into the following tableau in Fig. 10

$A$ carries on into $s$ and in $s$, we put $C$ on the left and $D$ on the right. ${ }^{4}$

This is the usual tableau process for intuitionistic logic. If we have $\wedge$ and $\vee$ in the language, we might get different alternatives (tableau splitting). Let us assume our language contains only $\rightarrow$ so that we can concentrate on the differences between ordinary tableaux and reactive tableaux, without the complexity generated by the presence of $\wedge$ and $\vee$.

In the case of pre-reactive semantics of Definition 2.3 the tableau will have labels $\alpha, \beta$ which are paths. So $t=\alpha$ and $s=\beta$. This is not an essential difference. The difference is essential in the reactive case because we must require that $\beta$ is a legitimate extension of $\alpha$. To do that we must record what $\mathbb{R}_{\beta}$ is.

So to simplify even further and allow us to present the essential ideas of the reactive tableau let us assume our logic has a fixed finite reactive frame, $(S, \mathbb{R}, a)$.

In this case we get the following simplifications:

(S1.) Since the frame is finite and for any legitimate sequence $\beta, \mathbb{R}_{\beta}$ is smaller than $\mathbb{R}$, there is only a finite number of frames $\left(S, \mathbb{R}_{i}\right), i=1, \ldots, n$, that are at play, (S2.) We can move to the finite folded Kripke frame $\mu=(\Omega, \rho, \approx, a)$ and do our tableaux on $\mu$. This is significantly simpler because $\mu$ is like an ordinary Kripke frame with the additional simple condition (simple from the tableaux point of view) imposed by $\approx$.

The next definition gives the notion of Beth tableaux for the implicational fragment. Note three facts:

Fact 1 Every wff can be put in the form

$$
E=\left[A_{1} \rightarrow\left(\ldots \rightarrow\left(A_{n} \rightarrow q\right) \ldots\right)\right]
$$

\footnotetext{
$4 A$ carries into $s$ because in an intuitionistic Kripke model we have that for any $A$ :$$
t \vDash A \text { and } t R s \rightarrow s \vDash A \text {. }
$$

Note that if for some reason we want that $t$ and $s$ agree on the values of atoms $q$, then any atom in the right hand side of $t$ will also carry to the right hand side of $s$. 
where $q$ is atomic and each $A_{i}$ is of the same form as $E$.

Fact 2 We need only two tableaux rules:

- To make $E$ false ( $E$ on the right) at world $t$, find a world $s$ such that $t \rho s$ and put all $A_{i}$ on the left and $q$ on the right.

- To make $E$ true on the left at $t$ when $q$ is on the right at $t$, we must move one of $A_{i}$ to the right of $t$ to make $A_{j}$ false.

Fact 3 To accommodate $\approx$ we make some adjustments to the usual intuitionistic tableaux rules.

Definition 4.1 (Beth tableaux for folded Kripke frames) Let $\mu=(\Omega, \rho, \approx)$ be a folded frame.

1. A tableau for $\mu$ is a pair of functions $\left(\tau^{+}, \tau^{-}\right)$from $\Omega$ into the set of wffs satisfying the following conditions:

(a) If $t \rho s$ holds in $\mu$ then $\tau^{+}(t) \subseteq \tau^{+}(s)$

(b) If $t \approx s$ holds then $\tau^{ \pm}(t)$ and $\tau^{ \pm}(s)$ contain the same atoms (respectively)

2. Let $\mathbf{T}$ be a family of tableaux $\tau$ as in (1). Let $\tau \in \mathbf{T}$. We define an operation which will split $\tau$ into several alternatives and we will replace $\tau$ in $\mathbf{T}$ by these alternatives to obtain a new family $\mathbf{T}^{\prime}$.

\section{Right hand operation}

Choose $t \in \Omega$ and choose $E$ in $\tau^{-}(t)$. Get the following alternatives $\tau_{s}^{ \pm}$to replace $\tau$ by $\left\{\tau_{s} \mid s \in \Omega\right.$ and $\left.t R s\right\}$ where

$$
\begin{aligned}
& \tau_{s}^{-}(x)= \begin{cases}\tau^{-}(x), & \text { for } x \not \approx s \\
t^{-}(x) \cup\{q\} & \text { for } x \approx s, x \neq t\end{cases} \\
& \tau_{s}^{-}(t)=\tau^{-}(t)-\{E\} \cup\{q\} \text { if } x=t \\
& \tau_{s}^{+}(x)= \begin{cases}\tau^{+}(x) & \text { if } x \not s s \\
\tau^{+}(x) \cup\left\{A_{i} \mid A_{i} \text { atomic }\right\} & \text { if } x \approx s \text { and } x \neq s \\
\tau^{+}(t) \cup\{A-i\} & \text { if } x=t\end{cases}
\end{aligned}
$$

\section{Left hand operation}

Choose $t \in \Omega$ and choose $E$ in $\tau^{+}(t)$ such that $q \in \tau^{-}(t)$.

Recall that $E=\left[A_{1} \rightarrow\left(\ldots \rightarrow\left(A_{n} \rightarrow q\right)\right)\right]$.

For each $A_{i}$ form the following tableau $\tau_{i}$.

$$
\begin{aligned}
& \tau_{i}^{+}(x)=\tau^{+}(x), x \in \Omega \\
& \tau^{-}(x)= \begin{cases}\tau^{-}(x) \cup\left\{A_{i}\right\}, & \text { if } A_{i} \text { is atomic and } x \approx t . \\
\tau^{-}(x) & \text { if } x \neq t \\
t^{-}(t) \cup\left\{A_{i}\right\} & \text { if } x=t .\end{cases}
\end{aligned}
$$

3. A tableau $\tau$ is closed if for some $t \in \Omega$ we have $\tau^{+}(t) \cap \tau^{-}(t) \neq \varnothing$.

4. $\mathbf{T}$ is closed iff all of its alternatives are closed.

Theorem 4.2 (Completeness) The above procedure is complete for the logic defined by the frame $\mu$. 
Proof Modify a proof for the case of an ordinary intuitionistic frame.

Definition 4.3 (Beth tableau for reactive Kripke frame) Let $\mathbf{m}=(S, \mathbb{R}, a)$ be a reactive frame. Let $\mu=(\Omega, \rho, \approx)$ be the folded frame effectively obtained from $\mathbf{m}$. Let $A$ be a wff for which we seek a counter model. Execute the algorithm of Definition 4.1. If an assignment $\mathbf{h}$ is found which falsifies $A$ in $\mu$, then the corresponding assignment $h$ is found which falsifies $A$ in $\mu$, then the corresponding assignment $h$ will falsify $A$ in $\mathbf{m}$. If the tableaux is closed and no such assignment exists, then no such assignment exists in $\mathbf{m}$. Thus the logic of $\mathbf{m}$ can be characterised using tableaux. Given a class $\mathbb{K}$ of finite reactive frames then the logic of $\mathbb{K}$ can be characterised as the intersection of the logics of its members. See Footnote 3.

\section{References}

Gabbay, D. Reactive Beth Tableaux for modal logics. April 2009, to appear in Annals of Mathematics in Artificial Intelligence 2011.

Gabbay, D. (2008). Reactive Kripke semantics and arc accessibility. In A. Avron, N. Dershowitz, \& A. Rabinovich (Eds.), Pillars of computer science: Essays dedicated to Boris (Boaz) Trakhtenbrot on the occasion of his 85th birthday (LNCS 4800, pp. 292-341). Berlin: Springer.

Gabbay, D., \& Marcelino, S. (2009). Modal logics of reactive frames. Studia Logica, 92, 403-444.

Maksimova, L. L. (1977). Craig's Theorem in superintuitionsitic logic and amalgamable varieties of pseudo-Boolean algebras. Algebra and Logic, 16, 427-455. 